\title{
Brain Metabolic Abnormalities Associated with Developmental Venous Anomalies
}

\author{
M. Larvie, D. Timerman, and J.A. Thum
}

\& $\mathrm{O}=$

\begin{abstract}
BACKGROUND AND PURPOSE: Developmental venous anomalies are the most common intracranial vascular malformation and are typically regarded as inconsequential, especially when small. While there are data regarding the prevalence of MR imaging findings associated with developmental venous anomalies, FDG-PET findings have not been well-characterized.
\end{abstract}

MATERIALS AND METHODS: Clinical information systems were used to retrospectively identify patients with developmental venous anomalies depicted on MR imaging examinations who had also undergone FDG-PET. Both the MR imaging and FDG-PET scans were analyzed to characterize the developmental venous anomalies and associated findings on the structural and functional scans. Qualitative and quantitative assessments were performed, including evaluation of the size of the developmental venous anomaly, associated MR imaging findings, and characterization of the FDG uptake in the region of the developmental venous anomaly.

RESULTS: Twenty-five developmental venous anomalies in 22 patients were identified that had been characterized with both MR imaging and FDG-PET, of which 76\% (19/25) were associated with significant metabolic abnormality in the adjacent brain parenchyma, most commonly hypometabolism. Patients with moderate and severe hypometabolism were significantly older (moderate: mean age, $65 \pm 7.4$ years, $P=.001$; severe: mean age, $61 \pm 8.9$ years, $P=.008$ ) than patients with developmental venous aberrancies that did not have abnormal metabolic activity (none: mean age, $29 \pm 14$ years).

CONCLUSIONS: Most (more than three-quarters) developmental venous anomalies in our series of 25 cases were associated with metabolic abnormality in the adjacent brain parenchyma, often in the absence of any other structural abnormality. Consequently, we suggest that developmental venous anomalies may be better regarded as developmental venous aberrancies.

ABBREVIATION: DVA = developmental venous anomaly

A developmental venous anomaly (DVA) is a transparenchymal vein of greater than usual size into which coalesces a network of smaller veins, a configuration described as a caput

Received July 31, 2014; accepted after revision September 18

From the Harvard Medical School (M.L.), Boston, Massachusetts; Divisions of Neuroradiology and Nuclear Medicine and Molecular Imaging (M.L., D.T., J.A.T.), Department of Radiology, Massachusetts General Hospital, Boston, Massachusetts; and Division of Health Sciences and Technology (D.T., J.A.T.), Harvard-Massachusetts Institute of Technology, Cambridge, Massachusetts.

This work was supported in part by the National Institutes of Health (P01 AG036694-01, 2R01 AG026484-06Al)

Paper previously presented in abstract form at: Annual Meeting of the American Society of Neuroradiology and the Foundation of the ASNR Symposium, May 1722, 2014; Montreal, Quebec, Canada.

Please address correspondence to Mykol Larvie, MD, PhD, Department of Radiology, Massachusetts General Hospital, 55 Fruit St, Boston, MA 02114; e-mail: mlarvie@gmail.com

- Indicates open access to non-subscribers at www.ajnr.org

三 Indicates article with supplemental on-line table.

http://dx.doi.org/10.3174/ajnr.A4172 medusa. DVAs may drain into either the superficial or deep venous systems and may occur in the cerebrum, cerebellum, brain stem, and spinal cord. DVAs are the most common intracranial vascular malformation, with a reported incidence of $2.6 \% .^{1,2}$ The mechanism of DVA formation is still controversial. The term "developmental venous anomaly" was first coined by Lasjaunias et $\mathrm{al}^{3}$ to emphasize their belief that these are embryologic variants of venous drainage rather than true vascular anomalies.

Brain parenchymal lesions have been widely reported in association with DVAs, with abnormalities apparent on angiography, ${ }^{4}$ $\mathrm{CT}^{5}$ and MR structural imaging, ${ }^{6-8}$ and functional imaging methods such as perfusion and diffusion-weighted imaging. ${ }^{9-12}$ Several case reports further exemplify how DVAs can be associated with clinical pathologies such as hemorrhagic transformation, ischemic complications, and epileptogenic foci. ${ }^{13-16}$ Recent studies also indicate the possible role of DVAs in the formation of cavernous malformations. ${ }^{9}$ These findings suggest that the clinical significance of abnormal brain anatomy and activity near a DVA 
is uncertain and has yet to be accurately evaluated. Furthermore, our clinical experience has revealed metabolic abnormality associated with DVAs, as determined by FDG-PET. These findings led us to the hypothesis that the metabolic activity in brain parenchyma in the region of a DVA is abnormal. In this study, we sought to better understand metabolic activity in the region of DVAs as assessed by FDG-PET.

\section{MATERIALS AND METHODS \\ Patient Population}

This study was performed at a tertiary care academic medical center for adult and pediatric patients and was approved by the institutional review board and ethics committee. A retrospective search for patients with DVAs identified on radiologic examinations during January 2000 to August 2013 at our institution was performed. The reports were analyzed for key terms by using an internal radiology report data base. There were 9337 radiology reports that included the phrases "developmental venous abnormality," "developmental venous anomaly," "venous angioma," "cerebral venous malformation," "cerebral venous medullary malformation," or "DVA" in the findings. Criteria for inclusion in the study were the following: 1) patients with at least 1 cerebral or cerebellar DVA identified by MR imaging and reconfirmed by a neuroradiologist (M.L.); 2) positive confirmation of the DVA, as defined by the presence of dilated medullary veins (caput medusa) converging to a large transparenchymal vein draining into a deep or superficial vein; and 3) availability of an FDG-PET scan performed within 1 year of the MR imaging examination demonstrating the DVA. Consequently, all included cases were initially identified and reported by a clinical staff radiologist and subsequently confirmed by a trained neuroradiologist for inclusion in this study. Exclusion criteria for this study were the following: 1) PET or MR imaging findings suggestive of a neurodegenerative disease; or 2) a confounding lesion, such as a tumor, in the region of the DVA or the anatomically equivalent contralateral cortex. One subject had a seizure at the time of PET imaging: This case is presented independently and was not included in the statistical data analysis of the correlates of hypometabolism. Of the $22 \mathrm{pa}-$ tients who qualified for this study, 25 DVAs were identified. The medical histories of the 22 patients were reviewed to identify demographic information, the initial reason for obtaining the imaging studies, and DVA characteristics (Table and On-line Table).

\section{MR Imaging}

The included MR imaging examinations were performed using a variety of imaging protocols that were prescribed according to the clinical indication for the scan. All MR imaging was performed at our institution using 1.5T (Signa, GE Healthcare, Milwaukee, Wisconsin; and Avanto, Siemens, Erlangen, Germany), 3T (Discovery, GE Healthcare; and Trio and Skyra, Siemens), and 7T (Siemens) scanners. When available, postcontrast T1-weighted imaging was used for the evaluation and confirmation of the DVA. In those cases in which postcontrast imaging was not available, sequences that reliably depicted flow voids, including T1and T2-weighted sequences, were used for evaluation of the DVAs. Any additional MR imaging sequences were also reviewed to assess the presence of additional findings in the area affected by

\begin{tabular}{lc}
\multicolumn{2}{c}{ Summary of characteristics of all patients and DVAs } \\
\hline \multicolumn{1}{c}{ Characteristic } \\
\hline Total No. of patients \\
No. of DVAs per patient \\
1 & 22 \\
2 & $19(86 \%)$ \\
Age (yr) (mean) & $3(14 \%)$ \\
Male, female sex & $41.5 \pm 19.8$ \\
DVA hemisphere: left, right & $11(50 \%), 11(50 \%)$ \\
Cerebral & \\
Cerebellar & $8(32 \%), 11(44 \%)$ \\
Indication for imaging patient & $2(8 \%), 4(16 \%)$ \\
Seizure & \\
Tumor & $11(50 \%)$ \\
Memory loss & $5(23 \%)$ \\
Infection & $1(5 \%)$ \\
Other & $1(5 \%)$ \\
Metabolic activity near DVA & $4(18 \%)$ \\
Isometabolic & \\
Hypometabolic & $6(24 \%)$ \\
Mild & $18(72 \%)$ \\
Moderate & $11(44 \%)$ \\
Severe & $4(16 \%)$ \\
Hypermetabolic & $3(12 \%)$ \\
Mild & $1(4 \%)$ \\
Moderate & $0(0 \%)$ \\
Severe & $0(0 \%)$ \\
\hline & $1(4 \%)$ \\
\hline DVA was con & \\
\hline
\end{tabular}

a Each DVA was counted separately in the 3 patients with multiple DVAs.

the DVA. The specific associated findings identified were the following: atrophy, calcification, hemorrhage, cavernous malformations, and abnormal T2 hyperintensity. Thirteen patients also had CT scans, which were reviewed to aid in the identification of calcification.

\section{FDG-PET Imaging}

All PET was performed at our institution using a standard protocol. Imaging was performed approximately 45 minutes following intravenous injection of $5.0-\mathrm{mCi}$ of $\left[{ }^{18} \mathrm{~F}\right]$ fluorodeoxyglucose by using an ECAT HR+ scanner (CTI-Seimens, Knoxville, Tennessee). Sixty-three planes were acquired simultaneously during a 15.5-cm FOV. Images were acquired in 3D mode. Attenuation correction was performed using a transmission scan obtained with a germanium-68 source. A maximum likelihood reconstruction method was used, yielding images with an in-plane resolution of approximately $4.6 \mathrm{~mm}$ (full width at half maximum). All patients were screened with fingerstick blood glucose measurement, and PET was not performed in those patients with blood glucose of $>150 \mathrm{mg} / \mathrm{dL}$.

\section{Image Analysis}

MR images and PET images were reviewed independently and coregistered using a PACS. PET images were also evaluated by comparison with normal standards to assess regional hypometabolism and hypermetabolism using internally developed software. FDG-PET findings in the brain parenchyma affected by DVAs was qualitatively evaluated and classified as normal, hypometabolic, or hypermetabolic in comparison with parenchyma in the contralateral brain hemisphere. Hypometabolism was further characterized as mild, moderate, and severe with respect to the range of findings in this study. This qualitative assessment of metabolism included both the extent of the lesion and the degree of met- 


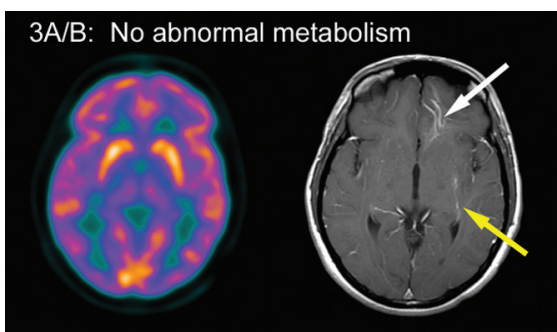

16: Moderate hypometabolism
10: Mild hypometabolism
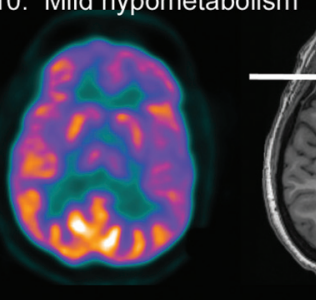

19: Severe hypometabolism

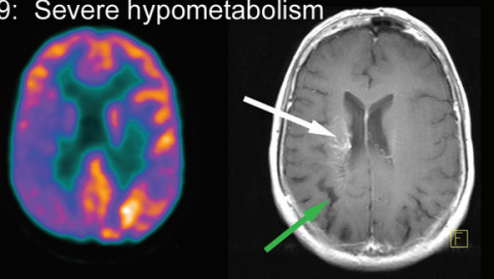

FIG 1. Representative FDG-PET and MR images demonstrating DVAs and associated metabolic activity. The numbering in the upper left of each image pair corresponds to the cases in the On-line Table. Cases 3A (white arrow) and 3B (yellow arrow) both do not demonstrate abnormal metabolism with respect to the contralateral cerebral hemisphere. Case 10 demonstrates mild hypometabolism in the anterior right frontal lobe corresponding to an area drained by a DVA. Case 16 demonstrates moderate hypometabolism in the posterior left frontal lobe corresponding to a DVA. Case 19 demonstrates severe hypometabolism distributed over the right frontal and parietal lobes and involving both cortical and deep gray matter in the region of a large DVA, which is not completely depicted on this single axial section. The green arrow in case 19 indicates atrophy.

abolic abnormality. We approximated the size of DVAs as the longest dimension on axial, sagittal, or coronal images, taking into account any visible feature of the DVA, including a caput medusa, if present, and the draining vein. This approximate measurement was obtained from the available imaging that best depicted the DVA, which was predominantly $\mathrm{T} 1$ postcontrast images, though other sequences that demonstrated the DVA were reviewed, including T1 precontrast, T2 and SWI, and T2* gradient recalledecho images. Both qualitative and quantitative assessments were assigned by the consensus of the 3 authors. Representative MR images and PET images were obtained of each DVA (Fig 1).

\section{Statistical Analysis}

The relationship between DVA size (in centimeters) and the qualitative degree of hypometabolism (normal, mild, moderate, or severe) was statistically analyzed for 24 cases. One of the 25 cases included in this study showed hypermetabolism in the clinical context of a seizure and was excluded from this analysis. Each of the datasets for the degree of hypometabolism was tested for equal variance. An ANOVA test was performed to determine whether the DVA sizes of the hypometabolic groups were significantly different, and pair-wise $t$ tests for equal variance among the 6 paired combinations of the 4 groups were performed to determine which groups had significantly different DVA sizes compared with each other. Differences with $P<.05$ were considered statistically significant.

\section{RESULTS}

Twenty-five DVAs were identified and characterized in 22 patients. A summary of the DVA characteristics and associated findings is presented in the On-line Table. Three patients each had 2 DVAs identified. The other 19 patients each had 1 DVA identified.

Clinical and demographic features of the patient cohort in this

study are presented in the Table. Eleven patients were male, and 11 were female; ages at the time of PET imaging ranged from 14 to 76 years (mean, $41.5 \pm 19.8$ years). Ten of the 25 DVAs (40\%) were identified on the patient's left side. Nineteen of the 25 DVAs (76\%) were supratentorial.

Among the 22 patients, the most common indications for imaging were seizure $(n=11,50 \%)$, tumor $(n=5$, $23 \%)$, memory loss ( $n=1,5 \%)$, infection ( $n=1,5 \%)$, and other (including paresthesias, motor deficits, and blurred vision; $n=4,18 \%$ ), as shown in the Table. At least 2 of the patients had symptoms that may have been due to the DVA. One of these patients had a DVA in the left perirolandic region that may have been a causative factor in the patient's reported right-sided paresthesias (case 16). Another patient (case 22) with a left temporo-occipital DVA had a seizure immediately following injection of FDG. The subsequent PET scan showed severe hypermetabolism associated with the DVA, which may reflect an underlying epileptogenic abnormality.

Qualitatively, 18 DVAs (72\%) exhibited some degree of hypometabolism, 6 DVAs (24\%) showed no visibly discernible metabolic difference between the cortical region drained by the DVA and the homologous contralateral brain, and 1 DVA (4\%) exhibited severe hypermetabolism (case 22, On-line Table).

Examples of the range of metabolic abnormalities seen in association with DVAs are shown in Fig 1, with the case numbering (top left of the image pairs) corresponding to the On-line Table. Cases $3 \mathrm{~A}$ and $3 \mathrm{~B}$ both demonstrate no abnormal metabolism in the region of the DVA, which is similar in metabolic activity to the contralateral hemisphere. Case $3 \mathrm{~A}$ is notable for a large DVA that has no associated metabolic abnormality. Cases 10, 16, and 19 demonstrate mild, moderate, and severe hypometabolism, respectively, compared with the contralateral cerebral hemisphere. Case 19 is a demonstration of hypometabolism involving both cortical and deep gray matter in the region of the DVA.

As shown in Fig 2, patients with DVAs that were associated with moderate and severe hypometabolism were significantly older (moderate: mean age, $65 \pm 7.4$ years, $P=.001$; and severe: mean age, $61 \pm 8.9$ years, $P=.008$ ) than patients with DVAs that were associated with normal metabolic activity (none: mean age, $29 \pm 14$ years). Furthermore, patients with mild hypometabolism were younger (mild: mean age, $33 \pm 16$ years) than those with moderate or severe hypometabolism, with $P=.002$ and $P=$ .013 for the respective comparisons.

As shown in Fig 3, DVAs that were associated with severe hypometabolism were significantly larger (mean, $5.0 \pm 1.5 \mathrm{~cm}$ ) than DVAs that were not associated with metabolic abnormality (mean, $2.3 \pm 0.6 \mathrm{~cm}, P=.011$ ) and were also significantly larger than DVAs with mild hypometabolism (mean, $2.4 \pm 1.0 \mathrm{~cm}, P=$ 


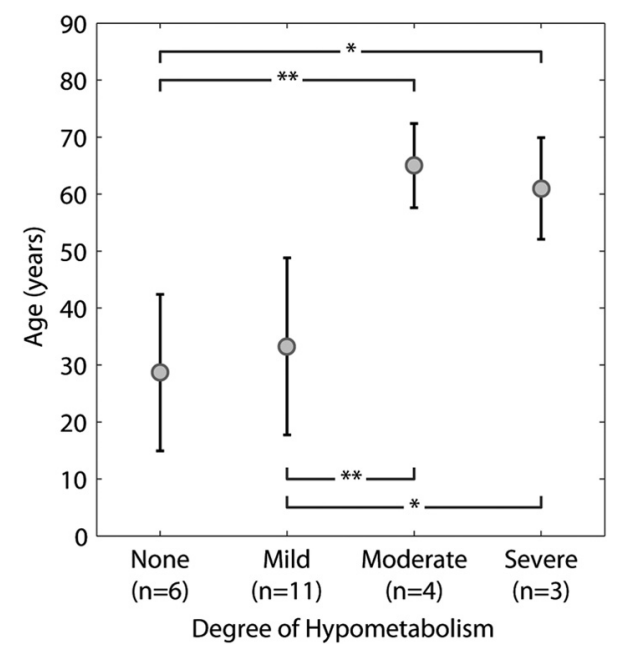

FIG 2. Relationship between age and degree of hypometabolism. Patients with DVAs with corresponding moderate and severe hypometabolism were significantly older (moderate: mean age, $65 \pm 7.4$ years, $P=$ .001 ; severe: mean age, $61 \pm 8.9$ years, $P=.008$ ) than patients with DVAs that were not associated with abnormal metabolism (none: mean age, $29 \pm 14$ years). Patients with a moderate $(P=.002)$ or severe $(P=.013)$ degree of hypometabolism were also older than patients with mild hypometabolism (mild: mean age, $33 \pm 16$ years). All patients who were 50 years or older $(n=8)$ had some degree of hypometabolism and included all cases with moderate $(n=4)$ and severe $(n=3)$ hypometabolism. A single asterisk indicates statistical significance at $P<.05$; double asterisks indicate statistical significance at $P<.005$. Error bars indicate 2 SDs.

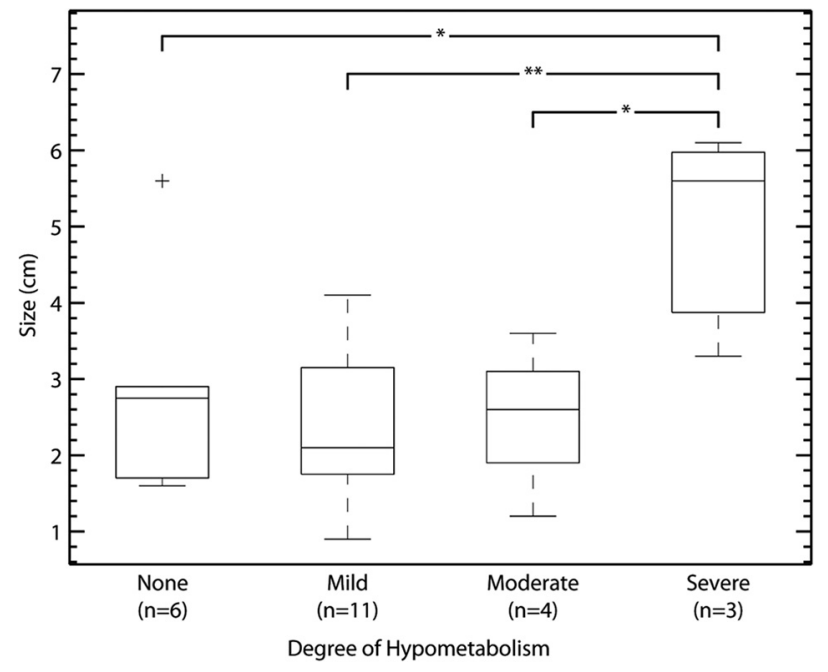

FIG 3. Size of the DVA versus the degree of hypometabolism in brain parenchyma drained by the DVA. DVAs associated with severe hypometabolism were larger (mean, $5.0 \pm 1.5 \mathrm{~cm}$ ) than those not associated with abnormal metabolism (mean, $2.3 \pm 0.6 \mathrm{~cm}, P=.011$ ), mild hypometabolism (mean, $2.4 \pm 1.0 \mathrm{~cm}, P=.003$ ), and moderate hypometabolism (mean, $2.5 \pm 1.0 \mathrm{~cm}, P=.043$ ). Case 3 A corresponds to the outlier, which was not included in the statistical analysis. This DVA was not associated with any degree of hypometabolism but had a large size measurement (indicated by a plus sign). DVA size was approximated by using the length of the single greatest dimension of the lesion. A single asterisk indicates statistical significance at $P<.05$; double asterisks indicate statistical significance at $P<.005$. Error bars indicate $2 \mathrm{SDs}$.

.003 ) and moderate hypometabolism (mean, $2.5 \pm 1.0 \mathrm{~cm}, P=$ .043). A statistically significant difference in DVA size was not observed between DVAs with no associated metabolic abnormality and those associated with mild or moderate hypometabolism.

The 1 DVA in our sample with corresponding hypermetabo- lism is depicted in Fig 4 and corresponds to case 22 in the On-line Table. In this case, a relatively small DVA is associated with severe hypermetabolism in the adjacent brain parenchyma.

Other findings identified in association with the DVAs were atrophy ( 4 cases), calcifications ( 2 cases, one a large calcification measuring $5.5 \mathrm{~cm}$ and the other a $1.5-\mathrm{cm}$ calcification), cavernous malformations (2 cases, both subcentimeter), hemorrhage (1 case), and elevated $\mathrm{T} 2$ hyperintensity (4 cases), as presented in the On-line Table. The hemorrhage, identified in a single case, was revealed by a $15-\mathrm{mm}$ arc of mild susceptibility signal in the deep white matter along the course of the DVA that was distinct from the associated flow void and was not apparent on a CT scan. This hemorrhage was within the deep white matter and was not clearly associated with hypometabolism in the adjacent cortex. The other findings-atrophy, calcifications, and cavernous malformations-were contained within the area of abnormal metabolism on the PET scan. Overall, 10 of the 25 total DVAs (40\%) were associated with a brain parenchymal lesion. Of the 24 nonhypermetabolic cases, all 6 DVAs that were scored as having normal metabolism in the cortex drained by the DVA had no additional abnormalities. Severe hypometabolism was observed in 1 of the 3 cases that exhibited atrophy, both of the 2 cases that had calcification, and 1 of the 4 cases with T2 hyperintensity. The 2 DVA cases with associated cavernous malformations also had corresponding hypometabolism.

\section{DISCUSSION}

We have undertaken this qualitative analysis to evaluate a relatively large number of cases with both structural imaging (principally MR imaging) and metabolic functional imaging with FDGPET. In this study, cortical hypometabolism associated with a DVA was observed in more than two-thirds of the 25 cases identified, supporting our hypothesis that the metabolic activity in the brain parenchyma in the region of a DVA is not necessarily definitively normal. This finding challenges the conventional understanding that DVAs are clinically inconsequential and do not affect adjacent brain parenchyma, ${ }^{3}$ though the significance of any metabolic abnormality remains uncertain.

The etiology of hypometabolism in the brain parenchyma drained by a DVA is unclear and may reflect an intrinsic, possibly developmental, neuronal abnormality or an acquired abnormality secondary to derangement of blood flow. We observed an association between the degree of DVA-associated hypometabolism and increased age of the patient. This association between hypometabolism in the drainage region of a DVA and age suggests the hypothesis that DVAs result in cumulative brain parenchymal injury. In this study, all 8 patients who were 50 years or older had some degree of hypometabolism, including all 4 cases with moderate hypometabolism and all 3 cases with severe hypometabolism.

DVAs have been documented in neonates, ${ }^{17,18}$ children, ${ }^{19}$ and adults. ${ }^{7}$ Risk stratification among age groups is challenging, given that the progression of brain abnormality and associated symptoms may be slow. One report showed that patients with signalintensity changes associated with a DVA were significantly older than those without. ${ }^{7}$

Our case series included a large DVA with no associated meta- 


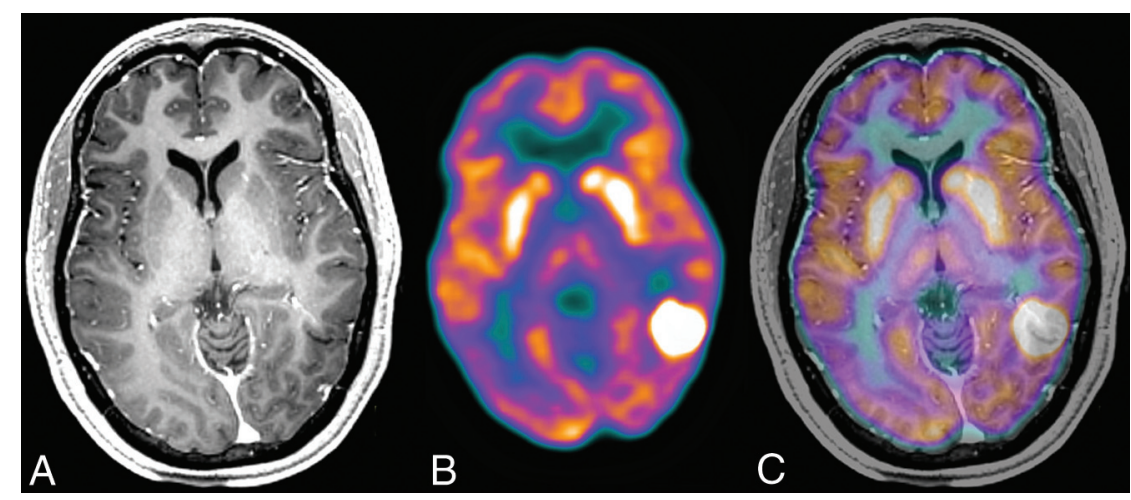

FIG 4. Case 22 is a patient who presented for evaluation qof epilepsy. MR imaging $(A)$ revealed a DVA in the left temporo-occipital region, seen here on postcontrast $\mathrm{Tl}$ images. Additional sequences (not shown) reveal elevated T2 signal in the region surrounding the DVA. The patient had seizure activity shortly after FDG administration, and FDG-PET (B) demonstrates focal severe hypermetabolism within the posterior left temporal lobe and anterior left occipital lobe that corresponds to the region of T2 hyperintensity on the MR imaging. Coregistration of the MR imaging and FDG-PET (C) demonstrates concordance between the PET abnormality and the DVA, suggesting the possibility that cortical abnormality related to the DVA may have resulted in an epileptogenic focus.

bolic abnormality (case 3A) in a 24-year-old patient. This stands as the single exception in this series, in which other large DVAs were associated with other brain parenchymal abnormalities. It would be informative to learn whether this lesion remains stable or additional brain parenchymal abnormalities develop with time.

Hemorrhagic or ischemic infarction around a DVA has been reported in multiple studies ${ }^{1,3,20}$ and has been attributed to acute thrombosis of the collecting vein. Not all patients with DVAs in our study had observable hypometabolism near the DVA, suggesting that either not all DVAs result in decreased metabolic activity or that infarction and subsequent brain parenchymal injury have yet to occur in such patients.

Few reports have used FDG-PET to evaluate the metabolic activity in the brain parenchyma drained by DVAs. In one case report, a patient presented with a DVA with associated increased FDG uptake in a region of MR imaging enhancement, which was attributed to "vascular changes, microcalcifications, and chronic inflammatory changes." ${ }^{20}$ Although it is challenging to appreciate the complete FDG-PET findings without a thorough analysis of the data, it appears that the axial map provided in Fig $2 \mathrm{~A}$ of that report could suggest marked hypometabolism associated with the DVA in the right cerebellum relative to the homologous contralateral region. In another case report, Planche et $\mathrm{al}^{21}$ described a patient with a DVA and cortical hypometabolism demonstrated on FDG-PET, though this finding was confounded by superimposed pathology of Sturge-Weber syndrome, including extensive pial angiomatosis.

Brain metabolism has been previously evaluated by using FDG-PET in patients with cavernous malformations. Ryvlin et $\mathrm{al}^{22}$ observed significantly reduced brain metabolism in 4 of 22 patients with cavernous malformations. Although DVAs are commonly associated with cavernous malformations, the 2 are distinct cerebral vascular malformations. Our finding of a higher incidence of metabolic abnormality associated with DVAs compared with cavernous malformations suggests that these vascular malformations have differential effects on the brain.
We observed 1 patient (case 22) who had seizure activity shortly after FDG administration, which manifested as extreme focal hypermetabolism in the posterior left temporal lobe, which corresponded to a T2 hyperintense cortical abnormality seen on the MR imaging (Fig 4). The association of DVAs and seizure has been previously reported ${ }^{23}$; however, to the best of our knowledge, this is the first report to observe hypermetabolic activity near the region of a DVA on FDG-PET. The concordance between the PET and MR imaging findings further suggests that DVAs may be epileptogenic. Alternatively, or in addition, the DVA in this case may be associated with a cortical malformation, which supports the hypothesis that DVA may be a marker of developmental abnormality that includes

both vessels and neurons.

The findings of this study have implications for the evaluation of patients with DVAs. We suggest that certain criteria may help to identify DVAs that could be symptomatic and that might warrant further evaluation, especially with FDG-PET. These include the following: 1) symptoms that could localize to the region of the DVA, 2) increased age (older than 50 years), and 3) large size ( $>4$ $\mathrm{cm}$ ). The presence or absence of brain parenchymal abnormalities on MR imaging was not strongly associated with metabolic abnormalities, so these findings may be less useful in the identification of symptomatic DVAs.

This study has several limitations. Although the number of DVAs included in this study is greater than that of any other DVA study evaluating FDG-PET findings, our collection of cases is relatively small owing to the relative scarcity of DVAs and the infrequency of FDG-PET examinations. Because the patients in this study underwent PET imaging as part of the inclusion criteria, there was a potential selection bias for patients with clinical symptoms that prompted the examination. Consequently, it is possible that the frequency with which hypometabolism is associated with DVAs is higher in our clinical population than in the general population. Nonetheless, the finding of hypometabolism in most cases reported here supports the conclusion that in some patients, the presence of a DVA correlates with and may cause brain parenchymal abnormality. Another limitation in this study is that the method used for measuring DVA size has limited accuracy in characterizing the overall extent of the lesion. DVAs are topologically diverse and not accurately characterized with orthogonal measurements. Furthermore, the PET findings in this study were qualitatively analyzed on the basis of visual inspection. A more quantitative volumetric analysis would better characterize the region of brain parenchyma affected by DVAs. Additionally, such a quantitative approach to the structural data would provide an improved approach to quantitative analysis of the PET data by providing a better defined region of interest for PET analysis.

AJNR Am J Neuroradiol 36:475-80 Mar 2015 www.ajnr.org 


\section{CONCLUSIONS}

We found that most (more than three-quarters) of the DVAs in our series of 25 cases were associated with metabolic abnormality in the adjacent brain parenchyma, as assessed by FDG-PET, often in the absence of any other structural abnormality. We also found that a more severe degree of DVA-associated hypometabolism was observed in older patients. In light of these findings, we suggest that DVAs may be better regarded as developmental venous aberrancies. A better understanding of the pathogenesis of the parenchymal metabolic abnormality associated with DVAs would be useful in the evaluation and possibly the management of patients with DVAs.

Disclosures: Mykol Larvie-RELATED: Grant: National Institutes of Health*; UNRELATED: Grants/Grants Pending: National Institutes of Health. * Money paid to the institution.

\section{REFERENCES}

1. Sarwar M, McCormick WF. Intracerebral venous angioma: case report and review. Arch Neurol 1978;35:323-25

2. Lee M, Kim MS. Image findings in brain developmental venous anomalies. J Cerebrovasc Endovasc Neurosurg 2012;14:37-43

3. Lasjaunias P, Burrows P, Planet C. Developmental venous anomalies (DVA): the so-called venous angioma. Neurosurg Rev 1986;9:233-42

4. Saito Y, Kobayashi N. Cerebral venous angiomas: clinical evaluation and possible etiology. Radiology 1981;139:87-94

5. Valavanis A, Wellauer J, Yaşargil MG. The radiological diagnosis of cerebral venous angioma: cerebral angiography and computed tomography. Neuroradiology 1983;24:193-99

6. Wilms G, Demaerel P, Robberecht W, et al. Coincidence of developmental venous anomalies and other brain lesions: a clinical study. Eur Radiol 1995;5:495-500

7. Santucci GM, Leach JL, Ying J, et al. Brain parenchymal signal abnormalities associated with developmental venous anomalies: detailed MR imaging assessment. AJNR Am J Neuroradiol 2008; 29:1317-23

8. Linscott LL, Leach JL, Zhang B, et al. Brain parenchymal signal abnormalities associated with developmental venous anomalies in children and young adults. AJNR Am J Neuroradiol 2014;35: 1600-07

9. Sharma A, Zipfel GJ, Hildebolt C, et al. Hemodynamic effects of developmental venous anomalies with and without cavernous malformations. AJNR Am J Neuroradiol 2013;34:1746-51

10. Jung HN, Kim ST, Cha J, et al. Diffusion and perfusion MRI findings of the signal-intensity abnormalities of brain associated with developmental venous anomaly. AJNR Am J Neuroradiol 2014;35: $1539-42$

11. Iv M, Fischbein NJ, Zaharchuk G. Association of developmental venous anomalies with perfusion abnormalities on arterial spin labeling and bolus perfusion-weighted imaging. J Neuroimaging 2014 Apr 9. [Epub ahead of print]

12. Matsuda $H$, Terada $T$, Katoh $M$, et al. Brain perfusion SPECT in a patient with a subtle venous angioma. Clin Nucl Med 1994:19: 785-88

13. Schneider SW, Ludwig T, Tatenhorst L, et al. Glioblastoma cells release factors that disrupt blood-brain barrier features. Acta Neuropathol 2004;107:272-76

14. Malik GM, Morgan JK, Boulos RS, et al. Venous angiomas: an underestimated cause of intracranial hemorrhage. Surg Neurol 1988;30:350-58

15. Nagatani K, Osada H, Takeuchi S, et al. Surgical resection of developmental venous anomaly causing massive intracerebral haemorrhage: a case report. Br J Neurosurg 2014;28:116-18

16. Griffiths D, Newey A, Faulder K, et al. Thrombosis of a developmental venous anomaly causing venous infarction and pontine hemorrhage. J Stroke Cerebrovasc Dis 2013;22:e653-55

17. Chang CL, Chiu NC. Developmental venous anomaly found by cranial US in a neonate. Pediatr Radiol 2010:40:374

18. Horsch S, Govaert P, Cowan FM, et al. Developmental venous anomaly in the newborn brain. Neuroradiology 2014;56:579-88

19. Gümüs A, Yildirim SV, Kizilkiliç O, et al. Case report: seizures in a child caused by a large venous angioma. J Child Neurol 2007;22: 787-89

20. Vaitkevicius H, Erdemli HE, Anderson WS, et al. Biopsy neuropathology of cerebral ischemia associated with a developmental venous anomaly. Clin Neurol Neurosurg 2013;115:621-23

21. Planche V, Chassin O, Leduc L, et al. Sturge-Weber syndrome with late onset hemiplegic migraine-like attacks and progressive unilateral cerebral atrophy. Cephalalgia 2014;34:73-77

22. Ryvlin P, Mauguière F, Sindou M, et al. Interictal cerebral metabolism and epilepsy in cavernous angiomas. Brain 1995;118(pt 3):677-87

23. Hon JM, Bhattacharya JJ, Counsell CE, et al. The presentation and clinical course of intracranial developmental venous anomalies in adults: a systematic review and prospective, population-based study. Stroke 2009;40:1980-85 